\title{
Inverse calculation of strain profiles from ETDR measurements using artificial neural networks
}

\author{
Robin Höhne, Pawel Kostka, and Niels Modler \\ Institute of Lightweight Engineering and Polymer Technology, Technische Universität Dresden, \\ Holbeinstraße 3, Dresden, Germany \\ Correspondence: Robin Höhne (robin.hoehne@tu-dresden.de)
}

Received: 29 August 2017 - Revised: 7 November 2017 - Accepted: 8 November 2017 - Published: 19 December 2017

\begin{abstract}
A novel carbon fibre sensor is developed for the spatially resolved strain measurement. A unique feature of the sensor is the fibre-break resistive measurement principle and the two-core transmission line design. The electrical time domain reflectometry (ETDR) is used in order to realize a spatially resolved measurement of the electrical parameters of the sensor. In this contribution, the process of mapping between the ETDR signals to the existing strain profile is described. Artificial neural networks (ANNs) are used to solve the inverse electromagnetic problem. The investigations were carried out with a sensor patch in a cantilever arm configuration. Overall, 136 experiments with varying strain distribution over the sensor length were performed to generate the necessary training data to learn the ANN model. The validation of the ANN highlights the feasibility as well as the current limits concerning the quantitative accuracy of mapping ETDR signals to strain profiles.
\end{abstract}

\section{Introduction}

In many structural health monitoring concepts strain gauges are commonly used. These strain gauges provide the required data for the prediction of the residual strength and durability of a component, such as a turbine blade or a car body part. In the case of complex shaped and loaded structures, the knowledge of spatially resolved strain fields is essential. Thus, there is a growing need of industry for spatially resolving strain sensors for structural health monitoring applications. In this context, current strain measurement systems like strain gauges or fibre optic sensors have some deficits regarding the complexity (multi-channel installation and processing), cost and design (weight and installation space). The challenge is to make a strain measurement system available that combines a high spatial resolution with manageable complexity, low weight and low costs. An innovative sensor technology has switched into the focal point of research at the Institute of Lightweight Engineering and Polymer Technology. A unique feature of the technology is the novel measurement principle based on carbon fibre fragments in combination with the electrical time domain reflectometry (ETDR). The interdisciplinary development of the sensor technology combines the knowledge of material science, production pro- cesses of fibre reinforced plastics and picosecond impulse technique. The synthesis of interdisciplinary findings makes the sensor technology a promising candidate to overcome current burdens of spatially resolved strain measurement. In this paper, both the measurement principle and the utilization of artificial neural networks (ANNs) from the field of machine learning, in order to measure the actual strain along the sensor in spatial resolution, are presented.

\subsection{Measurement principle of carbon fibre sensor}

The focused sensor patch basically represents a microstrip line, consisting of a brittle carbon fibre $(\mathrm{CF})$ roving and a copper strip which are embedded in a glass-fibre epoxy (GF-EP) carrier material and spaced by a GF-EP layer (see Fig. 1). The measurement principle was phenomenologically investigated in Höhne et al. (2017a) by means of microscopy images of the $\mathrm{CF}$ roving during tensile tests. The measurement principle is based on the reversible opening and closing of single filament fragments of the $\mathrm{CF}$ roving that were mechanically produced during the functionalization cycle. Thus the proposed strain sensor is based on a fibre-break resistive measurement principle enabling much higher resistance changes compared to conventional carbon fibre sensors us- 


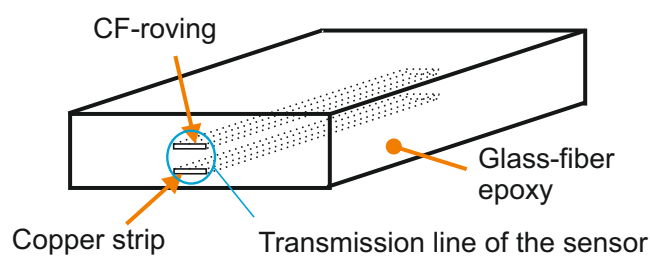

Figure 1. Schematic setup of the carbon fibre strain sensor showing the realization of the microstrip line embedded in a carrier material.

ing mainly the piezoresistive effect. Additionally, the integral electrical resistance of the sensor as a function of the homogeneous strain along the sensor was determined. A bijective, nonlinear function with progressive characteristic was identified. Furthermore, the endurance of the sensor was investigated in cyclic tests with swelling load, showing a stable functionality of up to $10^{6}$ cycles (Höhne et al., 2016).

The observed immense resistance changes of the CF roving result in a change of characteristic impedance of the microstrip. The characteristic impedance of a transmission line based on the telegrapher's equation in general is calculated as

$Z=\sqrt{\frac{R^{\prime}+\mathrm{j} \omega L^{\prime}}{G^{\prime}+\mathrm{j} \omega C^{\prime}}}$,

where $R^{\prime}$ is resistance, $L^{\prime}$ is inductance, $G^{\prime}$ is conductance and $C^{\prime}$ is capacitance. The apostrophe denotes the lengthspecific character. In the case of a single discontinuity (e.g. due to local strains and the associated resistance change), the reflection coefficient is

$\rho=\frac{E_{\mathrm{i}}}{E_{\mathrm{r}}}=\frac{Z_{\mathrm{L}}-Z_{0}}{Z_{\mathrm{L}}+Z_{0}}$,

where $E_{\mathrm{i}}$ and $E_{\mathrm{r}}$ are the voltage levels of incoming and reflected signal, respectively, and $Z_{\mathrm{L}}$ and $Z_{0}$ are the electrical impedances of two different sections of the transmission line. Due to the immense resistance change of the CF roving, the reflection coefficient is sufficiently high to be measured by means of the ETDR. The ETDR features a fast signal acquisition and potentially cheap and easy-to-miniaturize electronics with low energy consumption enabling handheld diagnostic units. The measurands in the ETDR are the reflections of an incident electrical wave at discontinuities of the characteristic impedance along the transmission line. In a typical application, a single measurement delivers a sampled sequence of up to several thousands of signal values depending on the spatially distributed properties of the transmission path. In Höhne et al. (2017b) the potential of the strain sensor for a spatially resolved strain measurement by means of the ETDR was highlighted. A spatial resolution capability in the low single-digit millimetre range was identified for a bending-loaded sensor.

From an electrotechnical point of view, a transmission line can be affected with hard and soft faults, whose identification is relevant for functionality purposes. Hard faults are open and short circuits, while soft faults are created by the change of impedance along the line due to different reasons, typically defects like insulation or deformation. Accordingly, the transmission line of the carbon fibre sensor is affected with soft faults due to the spatially distributed resistance change resulting from the fibre-break resistive measurement principle. The definition of the term "fault" has its origin in the field of electrical engineering, where any change of the tailored cable parameters adversely affects the functionality of the cable (e.g. high-efficient signal and energy transport). The term will be used in the following, although the change of impedance along the line is correctly viewed as not a fault in the case of the sensor application. In fact, it is an essential prerequisite for the measurement principle of the carbon fibre sensor with spatial resolution capability.

\subsection{Problem description}

In the preliminary investigations, the analysis of the sensor potential was limited to the unprocessed reflected voltage signal measured with the ETDR method. The measured signal cannot be directly associated with the strain distribution along the sensor, referred to as "strain profile" in the following. Advanced mathematical algorithms must be used for this purpose. The basic approach is to formulate an inverse electromagnetic problem (IEP). The IEP is typically ill-posed in the case of real measurements due to unavoidable (small) measurement errors resulting in an unpredictable effect on the solution (Oswald et al., 2003). The specific IEP of this investigation can be described as follows: determine a strain profile based on a known ETDR signal.

Several mathematical solution methods exist for solving IEP depending on the specific problem. Full waveform inversion (FWI) methods are frequently used in geoscience to solve the IEP for geotechnical site characterization, e.g. the estimation of the soil moisture (Schläger, 2005; Scheuermann et al., 2014) or water content (Oswald et al., 2003) along an ETDR sensing path. In this sensing application soft faults are considered as well. FWI methods use the full information content of the reflected ETDR signal to reproduce the spatial distribution of properties. It was shown that a high spatial resolution can be achieved with these FWI methods (Norgren and He, 1996). The applied FWI methods minimize a cost functional representing the deviation of the solution of the forward problem, calculated based on telegrapher's equations, and the measured ETDR signal. Calibration functions are additionally used to derive the focused physical parameters from the electrical parameters. Another iterative procedure based on genetic algorithms for detecting and localizing hard faults in complex wire networks can be found in Smail et al. (2010).

Both approaches require a large number of numerical calculations with the finite-difference method for each investigated signal profile in order to find the optimal solu- 
tion of the forward problem. The reported computing times range from several minutes (Schläger, 2005) to several hours (Baviskar and Heimovaara, 2017). This is disadvantageous for a large number of engineering-related measurement tasks, in particular for operando strain-based monitoring of complex lightweight structures. There may not be sufficient time to initiate safety measures in the case of potentially serious damage (e.g. due to a mechanical overload). Thus, the development of faster solution algorithms is a current need.

\subsection{Proposed Approach}

An alternative approach to solve the IEP is the use of ANNs as demonstrated in Coccorese et al. (1994). This approach features a significantly reduced calculation time, once the learning phase is completed. The (certainly time-consuming) learning of the ANN can advantageously be carried out before the usage of the sensor. During operation the inverse problem is solved very fast based on the trained ANN. In Smail et al. (2011) the performance of ETDR in combination with ANN in order to detect and localize hard faults (open circuits) in a wire network was highlighted. The possibility of extending the ANN method to the identification of soft faults is mentioned. In this case, the estimated parameters could be impedances.

In this context, the hypothesis is formulated that general statements on the corresponding strain profile can be made based on an ANN analysis of an ETDR signal of the described carbon fibre sensor. In order to map an ETDR signal to a strain profile, the linking of electrical and mechanical domain, from resistance to strain, is necessary. In preliminary investigations a bijective function describing the resistancestrain relation was determined (Höhne et al., 2017a, b) adding no further complexity to the mathematical task.

However, the goodness of the ANN results depends on the quality (variety and quantity) of the training data and the configuration of the neural network (e.g. the proper selection of the number of layers and neurons as well as a proper setting of weight and bias values during the learning process). The following conceptual process of establishing the ANN was used:

1. experimental generation of training data

2. ETDR signal processing (downsampling with antialiasing filtering)

3. design of ANN (define type, number of inputs, outputs, neurons and layers)

4. supervised learning of the ANN

5. validation of ANN (if required, resume with changed ANN design).

The scope of this work is to investigate the applicability of this approach and to determine the quantitative accuracy of the results.

\section{Experimental work}

\subsection{Test setup}

The test device introduced in Höhne et al. (2017b) was used for the experimental investigation of the spatially resolved strain measurement. A prototypical ETDR sensor patch according the configuration shown in Fig. 1 was clamped in a cantilever arm configuration (see Fig. 2a).

The sensor patch was connected to an ETDR device (DTDR-3000, Sympuls Aachen GmbH) using a coax cable with a characteristic impedance of $50 \Omega$. The inner and outer conductor of the coax cable were soldered to the copper strip and carbon fibre, respectively. The microstrip line of the sensor was terminated by means of a resistor matching the sensor impedance. As a result, no reflections $(r=0)$ occur at the end of the line that could complicate the signal analysis. Restrictive, however, it must be added that this is valid only for the unloaded condition of the sensor patch. The sensor impedance will change as soon as the patch is loaded due to the resistance change resulting from the patch strain. The sensor patch was loaded with a point load at the free end by hanging a weight. The maximum strain on the patch surface at the clamping is calculated as follows:

$\epsilon=\frac{m g l}{E I} e$,

where $m$ is the applied mass at the free end, $g$ is the gravitational constant, $l$ is the free bending length, $E$ is Young's modulus, $I$ is the moment of inertia of the patch cross section and $e$ is the distance of the CF roving to the bending neutral axis. In the case of a cantilever arm configuration, a linearly decreasing strain profile occurs at the sensor patch if a point load is applied at the free end (see Fig. 2b).

\subsection{Generation and preparation of training data}

The strain profile along the sensor patch can be precisely varied by shifting the sensor patch in the clamp and hanging different weights (Fig. 3a). Overall 136 experiments were performed with varying bending length and load in order to generate training data for the ANN. The bending length was varied stepwise in the range from 22 to $55 \mathrm{~mm}$ in $1 \mathrm{~mm}$ steps. For each bending length four load levels were applied by consecutively loading the free end of the sensor patch with no weight and weights of 78, 153 and $198 \mathrm{~g}$. The resulting strain profiles were calculated analytically according to Eq. (3) for these different configurations of free bending length and deflection. An overall maximum strain of approximately $0.3 \%$ at the clamping was realized in the investigated sensor/test device configuration. ETDR measurements were performed immediately after the load was applied.

The measured raw ETDR signal is composed of 501 values and is averaged over 10 single traces (Fig. 3b). In Höhne et al. (2017b) it was shown that the measured ETDR signal is 

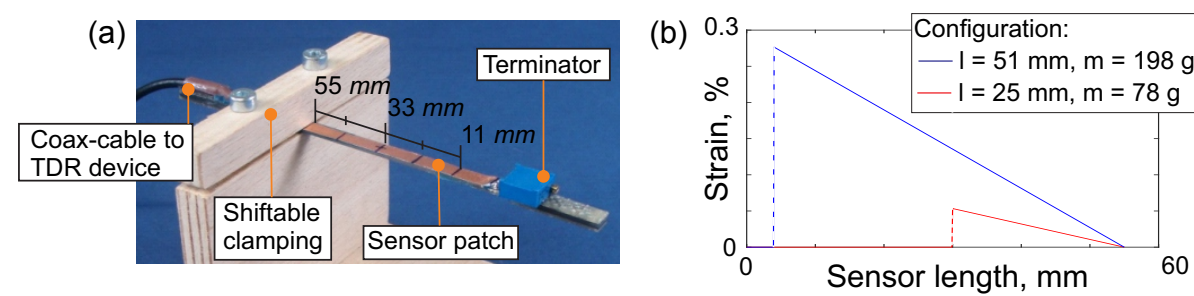

Figure 2. Clamped sensor patch in test device with a free bending length of $55 \mathrm{~mm}$ (a) and idealized strain profiles and an example shown for two configurations with different bending lengths and loading weights (b).

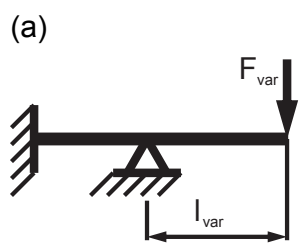

(b)

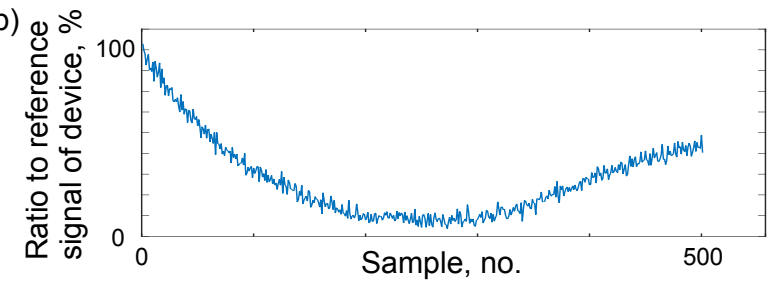

Figure 3. Conceptual configuration of different bending scenarios (a) and exemplary raw ETDR signal section used as training data (b).

a function of the free bending length. A spatial resolution in the low single-digit millimetre range for the investigated sensor configuration can be achieved. However, the measured ETDR signal cannot be directly associated with the existing strain profile so far.

\section{Design and learning of neural network}

The overall objective is to perform an ETDR-based spatially resolved strain measurement using the innovative carbon fibre sensor. Therefore a functional dependence between the known electromagnetical state of the sensor, expressed by the measured ETDR signal, and the spatially resolved mechanical strain of the sensor patch needs to be identified. This leads to an ill-posed, non-linear inverse electromagnetic problem. In this study, the solution approach comprises the application of ANN with a supervised learning procedure.

A feed-forward neural network model with 32 inputs, 8 outputs and an optimized number of 30 neurons in the hidden layer (HL) was designed. Several ANNs with different numbers of HL neurons and fixed inputs and outputs were learned using Levenberg-Marquardt algorithm. The procedure of solving the inverse problem is shown in Fig. 4. A single ETDR signal was supplied as input data to the model. The output data are then the retrieved strain profile. Each single input or output reflects a signal value of the ETDR signal or strain profile, respectively. Thus, the input signal has to be composed of 32 values whilst the output strain profile is a sequence of 8 values. Downsampling the ETDR signal from a sequence of 501 to 32 signal values was performed using signal filtering and re-picking of 32 equidistant grid points. Prior the use of the ANN a supervised learning has to be conducted. Therefore, $90 \%$ of the data sets were used as training data and the remaining data sets as validation data. During the learning process, the network's weight and bias values were updated according to Levenberg-Marquardt backpropagation algorithm. The finally selected ANN configuration represents the solution with the minimum error calculated for valid data. The described processing steps and the ANN setup were done using appropriate toolboxes of MATLAB (2017a).

\section{Results and discussion}

The ANN was validated in a hold-out procedure, i.e. on data sets resulting from configurations that were not used as training data. The results are shown for three exemplary configurations, covering the spectrum of adjustable strain profiles (Fig. 5). The results agree very well with the qualitatively characteristic of the analytically calculated strain profiles. However, the maximum relative errors between ANN estimated strain values and analytically calculated values scatter from 0.42 to $27.61 \%$ (Table 1 ). This can be attributed on the one hand to the limited number of used training data for the learning procedure and on the other hand to the fact that higher relative errors are observed for low strain values due to the relatively high noise level of the measuring data.

Since the generation of sufficient training data is a very time-consuming task and a high reproducibility of the experiment execution is required, an automated data generation process is recommended. This becomes even more important if more complex strain profiles are focused on than the investigated linear varying profiles. Such an automated data generation process could feature an improved time efficiency as well as increased quantity and quality of the training data. 
(a)

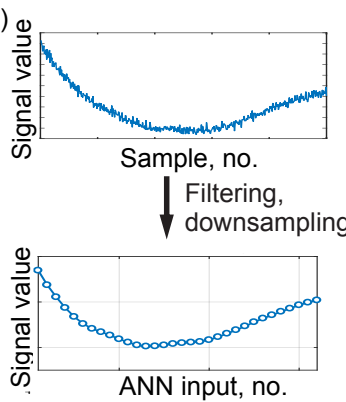

(b)

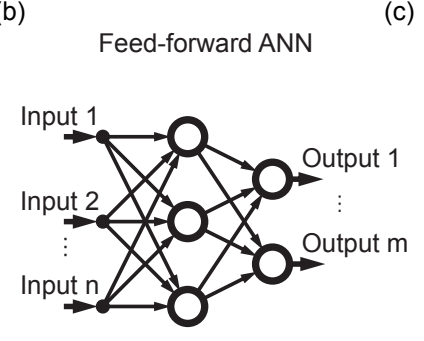

(c)

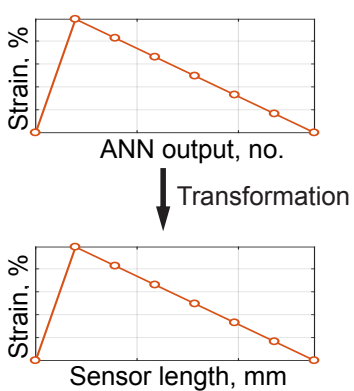

Figure 4. Procedure of the proposed mapping approach: processing of ETDR signal as ANN input data (a), conceptual design of the ANN (b), and interpretation of the ANN output as strain profiles (c).
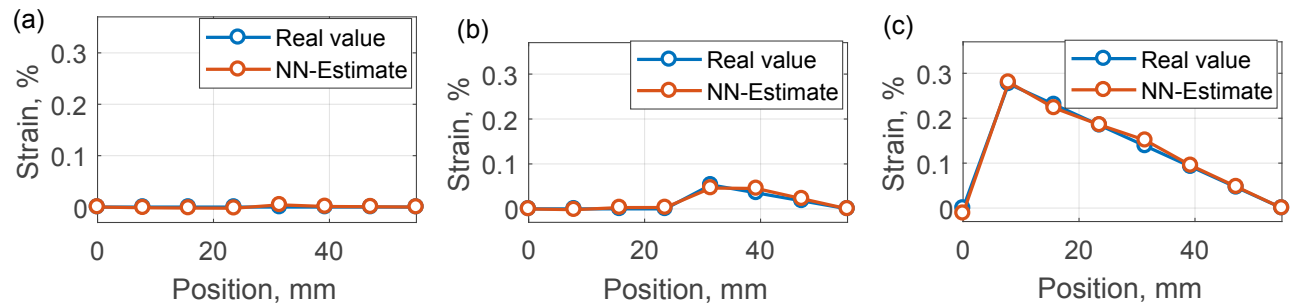

Figure 5. Results of the ANN model validation in bending test with no load and $40 \mathrm{~mm}$ possible bending length (a), a weight of $78 \mathrm{~g}$ and $25 \mathrm{~mm}$ bending length (b), and a weight of $198 \mathrm{~g}$ and $51 \mathrm{~mm}$ bending length (c).

Table 1. Absolute and relative error between analytical results and the ANN model estimation for different validation dates

\begin{tabular}{llrrrrrrrr}
\hline & & \multicolumn{1}{c}{ Grid point } \\
\cline { 3 - 9 } & Configuration & 1 & 2 & 3 & 4 & 5 & 6 & 7 & 8 \\
\hline \multirow{2}{*}{ Absolute error at grid point, ppm } & $25 \mathrm{~mm}, 78 \mathrm{~g}$ & -2.08 & -18.23 & 28.74 & 29.66 & -70.14 & 98.28 & 48.09 & 0 \\
& $51 \mathrm{~mm}, 198 \mathrm{~g}$ & -115.54 & 36.96 & -75.13 & 7.72 & 127.22 & 27.59 & 14.75 & 0 \\
\hline \multirow{2}{*}{ Relative error at grid point, \% } & $25 \mathrm{~mm}, 78 \mathrm{~g}$ & $*$ & $*$ & $*$ & $*$ & -13.14 & 27.61 & 27.02 & $*$ \\
& $51 \mathrm{~mm}, 198 \mathrm{~g}$ & $*$ & 1.34 & -3.26 & 0.42 & 9.20 & 2.99 & 3.20 & $*$ \\
\hline
\end{tabular}

* The relative error cannot be calculated since the real value is 0 .

This would add considerably value to the accuracy and robustness of the calculated strain profiles.

Furthermore it should be highlighted that the trained ANN provides the results with a very low numerical effort. The calculation time of a single strain profile of the focused complexity here is about $2 \mathrm{~ms}$ at a standard personal computer. The ANN approach could outperform the FWI methods, making it applicable not only for static measurements but also for dynamic strain measurements. A measurement frequency of $500 \mathrm{~Hz}$ can be realized for the investigated configuration. This requires an ETDR device that provides at least the same measurement frequency. Commercially available ETDR devices, however, are developed for static measurements due to the past and present needs. Thus, in order to exploit the full potential of the ANN approach for the solution of IEP, further development on the ETDR devices is also needed.

\section{Summary}

An innovative carbon fibre sensor with spatial resolution exploiting the low-cost and low-energy ETDR method for engineering applications has been developed. The spatial resolution capability requires the mapping of measured ETDR signals to existing strain profiles. The use of an ANN to solve the corresponding IEP was proposed and investigated.

A bending test device was used to generate the training data. Overall 136 experiments with different bending lengths and added weights were performed. An optimized feedforward ANN was established using a subset of 126 training sets. The subsequent ANN validation showed a qualitatively good agreement with the analytically calculated strain values. The observed quantitative errors can be explained by the limited number of training data used and the high noise amplitudes at the low strain values. Nonetheless, the results 
show the feasibility of mapping strain profiles to ETDR signals using ANNs. In addition, the ANN features short calculation time within milliseconds that could outperform the frequently used FWI methods by orders of magnitude. For more accurate results and especially if more complex strain profiles have to be identified, an automated sensor calibration method for learning the ANN should be established.

Data availability. No data sets were used in this article.

Competing interests. The authors declare that they have no conflict of interest.

Special issue statement. This article is part of the special issue "Sensor/IRS2 2017". It is a result of the AMA Conferences, Nuremberg, Germany, 30 May-1 June 2017.

Acknowledgements. The 3 Ccar project has received funding from ECSEL Joint Undertaking under grant agreement no. 662192. This Joint Undertaking received support from the European Union's Horizon 2020 research and innovation programme and Germany, Austria, Czech Republic, Romania, Belgium, United Kingdom, France, Netherlands, Latvia, Finland, Spain, Italy, and Lithuania.

Edited by: Stefan Rupitsch

Reviewed by: two anonymous referees

\section{References}

Baviskar, S. and Heimovaara, T.: Quantification of soil water retention parameters using multi-section TDR-waveform analysis, J. Hydrol., 549, 404-415, https://doi.org/10.1016/j.jhydrol.2017.03.068, 2017.

Coccorese, E., Martone, R., and Morabito, F. C.: A neural network approach for the solution of electric and magnetic inverse problems, IEEE T. Magn., 30, 2829-2839, https://doi.org/10.1109/20.312527, 1994.
Höhne, R., Kostka, P., and Modler, N.: Cyclic testing of novel carbon fiber based strain sensor with spatial resolution, 17th European Conference on Composite Materials, Munich, 2016.

Höhne, R., Ehrig, T., Kostka, P., and Modler, N.: Phenomenological investigation of a carbon fiber based strain sensor with spatial resolution by means of time domain reflectometry, Materialwiss. Werkst., 47, 1024-1033, 2017a.

Höhne, R., Kostka, P., and Modler, N.: Characterization of the spatial resolution capability of a novel carbon fiber strain sensor based on characteristic impedance measurements, Proceedings Sensor 2017, 166-171, 2017b.

MATLAB (2017a): MATLAB Neural Network Toolbox, the MathWorks, Natick, MA, USA, 2017.

Norgren, M. and He, S.: An optimization approach to the frequency-domain inverse problem for a nonuniform LCRG transmission line, IEEE T. Microw. Theory, 44, 1503-1507, https://doi.org/10.1109/22.536038, 1996.

Oswald, B., Benedickter, H. R., Bächtold, W., and Flühler, H.: Spatially resolved water content profiles from inverted time domain reflectometry signals, Water Resour. Res., 39, 1357, https://doi.org/10.1029/2002WR001890, 2003.

Scheuermann, A., Gonzales, C., Fan, J., and Braga, B.: Spatial Time Domain Reflectoemtry (spatial TDR) in geo-environmental engineering, Sensors Applications Symposium (SAS), Queenstown, New Zealand, 2014.

Schlaeger, S.: A fast TDR-inversion technique for the reconstruction of spatial soil moisture content, Hydrol. Earth Syst. Sci., 9, 481-492, https://doi.org/10.5194/hess-9-481-2005, 2005.

Smail, M. K., Pichon, L., Olivas, M., Auzanneau, F., and Lambert, M.: Detection of Defects in Wiring Networks Using Time Domain Reflectometry, IEEE T. Magn., 46, 2998-3001, https://doi.org/10.1109/TMAG.2010.2043720, 2010.

Smail, M. K., Hacib, T., Pichon, L., and Loete, F.: Detection and Location of Defects in Wiring Networks Using Time-Domain Reflectometry and Neural Networks, IEEE T. Magn., 47, 15021505, https://doi.org/10.1109/TMAG.2010.2089503, 2011. 